\title{
ÜBER EINIGE ALLGEMEINE KONVEXITÄTSSÄTZE IN DER THEORIE DER PARTIELLEN DIFFERENTIALGLEICHUNGEN
}

\author{
ALEXANDER DINGHAS
}

Herrn Kiyoshi Noshiro zum 60. Geburtstag

1. Einleitung. Es bezeichne $\boldsymbol{R}^{n}(n \geq 2)$ den durch den Alexandroff-Punkt kompaktifizierten $n$-dimensionalen euklidischen Raum, $\left[x_{1}, \ldots \ldots, x_{n}\right]$, ein Koordinatensystem von $\boldsymbol{R}^{n}, x=\left(x_{1}, \ldots \ldots, x_{n}\right)$ einen (endlichen) Punkt von $\boldsymbol{R}^{n}$ und

$$
\|x\|=\left(x_{1}^{2}+\ldots \ldots+x_{n}^{2}\right)^{\frac{1}{2}}
$$

dessen Norm. Wir betrachten ein im Endlichen liegendes Gebiet $G$ von $\boldsymbol{R}^{n}$, definieren auf $G$ einen linearen Operator

$$
L=\sum_{i, k=1}^{n} a_{i k} \frac{\partial^{2}}{\partial x_{i} \partial x_{k}}+\sum_{k=1}^{n} b_{k} \frac{\partial}{\partial x_{k}}+c \quad(c \leq 0)
$$

mit reellen, stetigen $a_{i k}=a_{i k}(x), b_{k}=b_{k}(x), c=c(x)^{1)}$ und bezeichnen bei festem $L$ durch $A_{0}[G]$ die Gesamtheit sämtlicher reellwertigen Lösungen $u$ der Differentialgleichung

$$
L[u]=\sum_{i, k=1}^{n} a_{i k} \frac{\partial^{2} u}{\partial x_{i} \partial x_{k}}+\sum_{k=1}^{n} b_{k} \frac{\partial u}{\partial x_{k}}+c u=0
$$

in $G$. Ist $O$ eine nicht leere Teilmenge von $G$, so soll allgemeiner $A_{0}[O]$ die Gesamtheit sämtlicher reellwertigen Funktionen $u \in C^{2}$, die auf $O$ definiert sind und der Differentialgleichung (1.2) genügen, darstellen.

Die Wahl des Operators $L$ wird im folgenden durch die Forderungen eingeschränkt:

(1) Das Dirichletsche Problem für die Differentialgleichung (1.2) ist für jedes Teilgebiet $G_{0}$ von $G$, das sich als Vereinigung von endlich vielen Kugeln darstellen läßt und dessen abgeschlossene Hülle $\bar{G}_{0}$ in $G$ liegt, eindeutig lösbar.

(2) Es gilt das (Hopfsche) Maximumprinzip in der Form:2)

Es bezeichne $u$ eine reellwertige Funktion mit den Eigenschaften: 
1. $u$ ist stetig in $\bar{G}_{0}$.

2. Auf $\Gamma_{0}=\bar{G}_{0}-G_{0}$ gilt $u \leq 0$.

3. $u$ ist zweimal stetig differenzierbar in $G_{0}$ und genügt dort der Ungleichung $L[u] \geq 0$.

Dann ist $u$ nicht positiv in $G_{0}{ }^{3}$ )

Die Bedingungen (1) und (2) sind offenbar stets erfüllt, wenn der Operator $L$ elliptisch ist und in $G$ die Ungleichung $c \leq 0$ gilt.

Die Funktionenklasse $A_{0}[G]$ kann durch die Familie $A[G]$ sämtlicher (in bezug auf (1.2)) subharmonischen Funktionen mit den Bedingungen ergänzt werden:

(i) $u \in A[G] \Longrightarrow-\infty \leqq u(x)<\infty, u(x) \not \equiv-\infty$

in $G$.

(ii) $u \in A[G] \Longrightarrow$

$$
\varlimsup_{y \rightarrow x} u(y) \leq u(x) \quad(\forall x \in G)
$$

(Halbstetigkeit nach oben).

(iii) Es bezeichne $v$ eine Lösung von (1.2) in $G_{0}$ mit den (stetigen) Randwerten $v^{-}$auf $\Gamma_{0}=\bar{G}_{0}-G_{0}$. Dann folgt aus $u(x)-v(x) \leq 0\left(x \in \Gamma_{0}\right)$ stets $u(x) \leq v(x)\left(x \in G_{0}\right)_{.4}^{4}$

Die Definition der Klassen $A_{0}[O]$ bzw. $A[O]$ für eine offene (nicht leere) Teilmenge $O$ von $G$ verläuft analog.

Folgendes Ergebnis ist für die nachfolgenden Entwicklungen von grundlegender Bedeutung:

Es sei $O$ eine nicht leere Teilmenge von $G$ und $\Gamma$ der Rand von $O$. Gibt es dann bei gegebenem $u \in A[G]$ ein $v \in A_{0}[O]$ mit der Eigenschaft

$$
\varlimsup_{x \rightarrow \Gamma}[u(x)-v(x)] \leq 0,
$$

so gilt $u(x) \leq v(x)$ in $O$. Dabei bedeutet die Schreibweise $x \rightarrow \Gamma$, daß der Punkt $x$ von $O$ auf irgendeine Weise gegen den Rand $\Gamma$ von $O$ konvergiert. Es bezeichne in der Tat $O_{1}$ eine (fest gewählte) Komponente von $O$ und $\Gamma$ den Rand von $O_{1}$. Man setze $\psi=u-v$ und nehme an, die Größe

$$
p=\sup _{x \in O_{1}} \psi(x)
$$

sei positiv. Dann gibt es mit Rücksicht auf die Halbstetigkeit von $\psi$ in $O_{1}$ einen Punkt $x_{0}$ von $O_{1}$ mit $\psi\left(x_{0}\right)=p$. Es sei nun $0<\varepsilon<p$. Dann gibt es ein 
$G_{0}$ mit den Eigenschaften: (i) $x_{0} \in G_{0}$, und (ii) $\psi \leq \varepsilon$ auf dem Rand $\Gamma_{0}$ von $G_{0}$. Man konstruiere die Lösung $v_{0}$ von (1.2) in $G_{0}$, die auf $\Gamma_{0}$ die Werte 1 annimmt, und beachte, daß wegen $\psi \in A\left[O_{1}\right]$ die Ungleichung $\psi(x) \leq \varepsilon v_{0}(x)$ in $G_{0}$ geltèn muß. Andererseits gilt neben $v_{0}=1$ auf $\Gamma_{0}$ noch

$$
L\left[v_{0}-1\right]=L\left[v_{0}\right]-L[1]=-c \geq 0
$$

in $G_{0}$ Das hat die Ungleichung $v\left(x_{0}\right) \leq 1$ zur Folge, die jedoch mit der Voraussetzung $\varepsilon<p=\psi\left(x_{0}\right) \leq \varepsilon v_{0}\left(x_{0}\right) \leq \varepsilon$ unvereinbar ist. Es ist also $p \leq 0$ und somit $\psi \leq 0$ in $O_{1}$. Die Wiederholung des Beweisverfahrens für jede Komponente von $O$ beweist die Ungleichung $u(x) \leq v(x)$ in $O$.

Neben $A[G]$ spielt bei den nachfolgenden Entwicklungen die Klasse der in $G$ superharmonischen Funktionen eine (duale) Rolle. Diese wird durch die Gleichung

$$
A^{\prime}=\{u:-u \in A[G]\}
$$

definiert.

Die vorliegende Arbeit hat zum Hauptzweck, eine Reihe von allgemeinen Konvexitätssätzen für die Klasse $A[G]$ zu beweisen, sofern die Existenz bestimmter singulärer Lösungen von (1.2) in $G$ vorausgesetzt wird. Diese Lösungen existieren mindestens für spezielle Randmengen, falls (1.2) die Form

$$
\Delta u=\frac{\partial^{2} u}{\partial x_{1}^{2}}+\ldots \ldots+\frac{\partial^{2} u}{\partial x_{n}^{2}}=0
$$

hat und $G$ eine Kugel um den Nullpunkt von $R^{n}$ ist. Die Differentialgleichung (1. 6) ist insofern von Interesse, als sich, wie später gezeigt werden soll, eine Reihe grundlegender Sätze der Funktionentheorie als Spezialfall eines allgemeinen Satzes erweisen lassen.

Die Ergebnisse dieser Arbeit, soweit sie allgemeiner Natur sind, können in geeigneter Formulierung ohne Schwierigkeit auf Mannigfaltigkeiten übertragen werden, sofern die Funktionen $a_{i k}$ und $b_{k}$ ein in bezug auf Transformationen des (lokalen) Koordinatensystems bestimmtes kovariantes Verhalten aufweisen. Doch sollen die diesbezüglichen Entwicklungen auf eine spätere Zeit verschoben werden.

2. Vorbereitende Tatsachen. Es bezeichnen $G$ ein (nicht leeres) Gebiet von $\boldsymbol{R}^{n}$ und

$$
f_{1}=\left[f_{1}(x)\right], \quad f_{2}=\left[f_{2}(x)\right]
$$


zwei auf $G$ definierte reellwertige stetige Funktionen mit den Eigenschaften 1.

$$
\left.f_{2}>0 \quad \text { (d.h. } f_{2}(x)>0, \quad \forall x \in G\right) .
$$

2. Die Funktion

$$
\omega=[\omega(x)]=\left[\frac{f_{1}(x)}{f_{2}(x)}\right]
$$

ist in $G$ nicht konstant.

Definition 1. Bei gegebenem $G$ wird gesetzt:

1.

2.

3.

4 .

5.

6.

7.

8.

9.

$$
\begin{aligned}
& a=\inf _{x \in G} \omega(x) . \\
& b=\sup _{x \in G} \omega(x) .
\end{aligned}
$$

$$
J=] a, b[, \quad \bar{J}=[a, b] .
$$$$
\Gamma_{\sigma}=\{x: x \in G, \quad \omega(x)=\sigma, \sigma \in J\} .
$$$$
O_{\sigma_{1}}^{\sigma_{2}}=\left\{x: x \in G, \sigma_{1}, \sigma_{2} \in J, \quad \sigma_{1}<\sigma_{2}, \sigma_{1}<\omega(x)<\sigma_{2}\right\} .
$$

$$
\bar{O}_{\sigma_{1}}^{\sigma_{2}}=O_{\sigma_{1}}^{\sigma_{2}} \cup \Gamma_{\sigma_{1}}^{\sigma_{2}}=\left\{x: x \in G, \sigma_{1} \leq \omega(x) \leq \sigma_{2}\right\} .
$$$$
\Gamma_{\sigma_{1} \sigma_{2}}=\bar{O}_{\sigma_{1}}^{\sigma_{2}} \cap \Gamma(\Gamma=\bar{G}-G=\mathrm{Rand} \text { von } G) \text {. }
$$

$$
\Gamma_{a}=\bigcap_{\sigma}\left\{\bar{O}_{a}^{\sigma}: \sigma \in J\right\} .
$$

$$
\Gamma_{b}=\bigcap_{\sigma}\left\{\bar{O}_{\sigma}^{b}: \sigma \in J\right\} .
$$

Definition 2. Es bezeichne $f=[f(x)]$ eine numerische, nach oben halbstetige Funktion auf G. Man setze für $\sigma \in J$

$$
\mu(\sigma, f)=\sup _{x \in \Gamma_{\sigma}} f(x)
$$

Dann soll $\mu=[\mu(\sigma, f)]$ konvex in $J$ heißen, wenn für jedes Wertetripel $\left(\sigma_{1}, \sigma, \sigma_{2}\right)$ mit $\sigma_{1}<\sigma<\sigma_{2}$ die Ungleichung

$$
\mu \leq \mu_{1} \frac{\sigma_{2}-\sigma}{\sigma_{2}-\sigma_{1}}+\mu_{2}-\frac{\sigma-\sigma_{1}}{\sigma_{2}-\sigma_{1}}
$$

gilt. Dabei sollen $\mu, \mu_{1}, \mu_{2}$ die Größen $\mu(\sigma, f), \mu\left(\sigma_{1}, f\right), \mu\left(\sigma_{2}, f\right)$ bedeuten.

Ist $h=[h(\sigma)]$ eine in $J$ stetige monotone Funktion und definiert man $\Gamma_{\sigma}$ durch die Gleichung

$$
\Gamma_{\sigma}=\left\{x: x \in G, h^{-1}(\omega(x))=\sigma, \sigma \in J\right\}
$$


so gilt anstelle (2.3) die Ungleichung

$$
\mu \leq \mu_{1} \frac{h_{2}-h}{h_{2}-h_{1}}+\mu_{2} \frac{h-h_{1}}{h_{2}-h_{1}}
$$

mit $h_{1}=h\left(\sigma_{1}\right), h_{2}=h\left(\sigma_{2}\right), h=h(\sigma)$. In diesem Falle soll $\mu h$-konvex in $J$ heißen.

Gilt

$$
\mu \geq \mu_{1} \frac{\sigma_{2}-\sigma}{\sigma_{2}-\sigma_{1}}+\mu_{2} \frac{\sigma-\sigma_{1}}{\sigma_{2}-\sigma_{1}}
$$

in $J$, so heißt $\mu$ konkav in $J$. Entsprechend soll $\mu h$-konkav in $J$ heißen, wenn dort (unter Zugrundelegung von (2. 4))

$$
\mu \geq \mu_{1} \frac{h_{2}-h}{h_{2}-h_{1}}+\mu_{2} \frac{h-h_{1}}{h_{2}-h_{1}}
$$

gilt.

Ist $\mu$ konvex in $J$, so ist offenbar $-\mu$ konkav in $J$.

SAtz 1. Es sei $a=0$ und $b=+\infty$. Dann hat jede in $J$ konvexe Funktion $\mu=[\mu(\sigma, f)]$ die Eigenschaften:

(1) Es existieren die Grenzwerte

$$
A_{0}=A_{0}(f)=\lim _{\sigma \rightarrow 0} \mu(\sigma, f) \quad\left(-\infty<A_{0} \leq+\infty\right)
$$

und

$$
A_{\infty}=A_{\infty}(f)=\lim _{\sigma \rightarrow+\infty} \frac{\mu(\sigma, f)}{\sigma} \quad\left(-\infty<A_{\infty} \leq+\infty\right) .
$$

(2) Es ist

$$
\mu(\sigma, f) \leq A_{0}+A_{\infty} \sigma .
$$

Beweis. Aus (2.3) erhält man zunächst

$$
\frac{\mu}{\sigma} \leq \frac{\mu_{1}}{\sigma_{1}} \frac{1-\frac{\sigma_{2}}{\sigma}}{1-\frac{\sigma_{2}}{\sigma_{1}}}+\frac{\mu_{2}}{\sigma_{2}} \frac{1-\frac{\sigma_{1}}{\sigma}}{1-\frac{\sigma_{1}}{\sigma_{2}}} .
$$

Man setze

$$
\underline{A}_{\infty}=\lim _{\sigma \rightarrow+\infty} \frac{\mu}{\sigma}, \bar{A}_{\infty}=\varlimsup_{\sigma \rightarrow+\infty} \frac{\mu}{\sigma} .
$$

Dann folgt aus 


$$
\frac{\mu}{\sigma} \leq \frac{\mu_{1}}{\sigma}+\underline{A} \infty\left(1-\frac{\sigma_{1}}{\sigma}\right)
$$

also auch (durch Grenzübergang $\sigma \rightarrow+\infty) \bar{A}_{\infty} \leq \underline{A}_{\infty}$. Somit wird

$$
\mu \leq \mu_{1}+A_{\infty}\left(\sigma-\sigma_{1}\right) .
$$

Andererseits folgt aus (2.3) bei entsprechender Definition von $\underline{A}_{0}, \bar{A}_{0}$

$$
\mu \leq \underline{A}_{0}\left(1-\frac{\sigma}{\sigma_{2}}\right)+\mu_{2} \frac{\sigma}{\sigma_{2}}
$$

und somit auch $\bar{A}_{0} \leq \underline{A}_{0}, d . h . \underline{A}_{0}=\bar{A}_{0}=A_{0}$. Das liefert mit Rücksicht auf die Ungleichung (2.12) die Ungleichung (2.10).

Auf ähnliche Weise beweist man den zum Satz 1 dualen Satz:

SATz 2. Ist $\mu$ konkav in $J$ und $a=0, b=+\infty$, so existieren die Grenzwerte

$$
B_{0}=B_{0}(f)=\lim _{\sigma \rightarrow 0} \mu(\sigma, f) \quad\left(-\infty \leqq B_{0}<+\infty\right)
$$

und

$$
B_{\infty}=B_{\infty}(f)=\lim _{\sigma \rightarrow+\infty} \frac{\mu(\sigma, f)}{\sigma} \quad\left(-\infty \leqq B_{\infty}<+\infty\right)
$$

und es gilt die Ungleichung

$$
\mu \geq B_{0}+B_{\infty} \sigma .
$$

Die Ungleichungen (2.10) und (2.16) sind offenbar dann von Bedeutung, wenn die Größen $A_{0}, A_{\infty}, B_{0}, B_{\infty}$ endlich sind.

3. Erste Konvexitätssätze. Beim Versuch, allgemeine Konvexitätssätze für die Differentialgleichung (1.2) aufzustellen und $\mathrm{zu}$ beweisen, wird man auf Fragestellungen geführt, deren Beantwortung die Existenz von zwei grundsätzlich verschiedenen singulären Lösungen erfordert. Die Lösungen hängen wiederum mit der Aufgabe der Ausschöpfung eines gegebenen Gebiets $G$ von $\boldsymbol{R}^{n}$ durch geeignete, durch das Problem selbst bedingte, Umgebungssysteme zusammen, sowie mit der Lokalisierung von Randmengen von $G$ durch solche Systeme. Insbesondere führt hier die zweite dieser Fragestellungen, sofern man sie weiter vertiefen will, zu Problemen, die der Theorie der Primenden von CARATHÉODORY mehr oder weniger untergeordnet sind.

SATz 3. Es bezeichnen $u_{1}, u_{2}$ zwei Lösungen von (1. 2) in $G$ mit den Eigenschaften: 1. $u_{2}>0$. 
2. Die Funktion $\omega=\frac{u_{1}}{u_{2}}$ ist in $G$ nicht konstant.

3. Es gilt

$$
\lim _{x \rightarrow \Gamma} u_{2}(x)=1 \quad(\Gamma=\text { Rand von } G)^{5)} .
$$

4. Die Randmengen $\Gamma_{a}, \Gamma_{b}$ sind disjunkt, und es gilt

$$
\lim _{x \rightarrow \Gamma_{a}} \omega(x)=a, \lim _{x \rightarrow \Gamma_{b}} \omega(x)=b
$$

und $\Gamma=\Gamma_{a} \cup \Gamma_{b}$.

Man setze bei gegebenem $u \in A[G]$

$$
M\left(\sigma, \frac{u}{u_{2}}\right)=\max _{x \in \Gamma_{\sigma}} \frac{u(x)}{u_{2}(x)} .
$$

Dann ist $M\left(\sigma, \frac{u}{u_{2}}\right)$ konvex in $\left.J=\right] a, b[$.

Beweis. Man schreibe $M_{1}, M, M_{2}$ für $M\left(\sigma_{1}, \frac{u}{u_{2}}\right), M\left(\sigma, \frac{u}{u_{2}}\right), M\left(\sigma_{2}, \frac{u}{u_{2}}\right)$ und bilde die Funktion

$$
v=u-\left\{M_{1} \frac{\sigma_{2}-\omega}{\sigma_{2}-\sigma_{1}}+M_{2} \frac{\omega-\sigma_{1}}{\sigma_{2}-\sigma_{1}}\right\} u_{2}
$$

in $O_{\sigma_{1}}^{\sigma_{2}}$. Dann liegt zunächst $v$ wegen

$$
v=u-\frac{M_{2}-M_{1}}{\sigma_{2}-\sigma_{1}} u_{1}+\frac{M_{2} \sigma_{1}-M_{1} \sigma_{2}}{\sigma_{2}-\sigma_{1}} u_{2}
$$

in $A\left[O_{\sigma_{1}}^{\sigma_{2}}\right]$. Andererseits folgt aus (3.4) bei Annäherung an den Rand $\Gamma_{\sigma_{1}}^{\sigma_{2}}$ von $O_{\sigma_{1}}^{\sigma_{2}}$

$$
\varlimsup_{x \rightarrow \Gamma_{\sigma_{1}}^{\sigma_{2}}} \frac{v(x)}{u_{2}(x)} \leq 0
$$

und somit auch

$$
\varlimsup_{x \rightarrow \Gamma_{\sigma_{1}}^{\sigma_{2}}} v(x) \leq 0
$$

Das liefert (wegen $v \in A\left[O_{\sigma_{1}}^{\sigma_{2}}\right]$ ) die Ungleichung $v(x) \leq 0$ in $O_{\sigma_{1}}^{\sigma_{2}} d . h$.

$$
u(x) \leq\left\{M_{1} \frac{\sigma_{2}-\omega(x)}{\sigma_{2}-\sigma_{1}}+M_{2} \frac{\omega(x)-\sigma_{1}}{\sigma_{2}-\sigma_{1}}\right\} u_{2}(x)
$$


in $O_{\sigma_{1}}^{\sigma_{2}}$. Die Einschränkung von $x$ auf $\Gamma_{\sigma}$ liefert ohne weiteres die Ungleichung

$$
M \leq M_{1} \frac{\sigma_{2}-\sigma}{\sigma_{2}-\sigma_{1}}+M_{2} \frac{\sigma-\sigma_{1}}{\sigma_{2}-\sigma_{1}}
$$

und somit den Beweis des Satzes 3.

SATz 4. Es bezeichnen $u_{1}, u_{2}$ zwei Lösungen von (1. 2) mit den Eigenschaften:

1.

$$
u_{1}, u_{2}>0 \text {. }
$$

2.

$$
\lim _{x \rightarrow \Gamma} u_{2}(x)=1 \quad(\Gamma=\bar{G}-G) \text {. }
$$

3.

$$
a=\inf _{x \in G} \omega(x)=\inf _{x \equiv G} \frac{u_{1}(x)}{u_{2}(x)}=0 .
$$

4.

$$
b=\sup _{x \in G} \omega(x)=\sup _{x \in G} \frac{u_{1}(x)}{u_{2}(x)}=+\infty .
$$

5.

$$
\lim _{x \rightarrow x_{1}} u_{1}(x)=0 \quad\left(\forall x_{1} \in \Gamma-\Gamma_{\infty}\right) .
$$

6.

$$
\bar{O}_{\sigma_{2}}^{\sigma_{2}} \cap \Gamma=\Gamma_{\infty} \quad(\forall] \sigma_{1}, \sigma_{2}[\subseteq J=] 0,+\infty[) .
$$

Es sei $u \in A[G]$ und habe die Eigenschaft

$$
\lim _{x \rightarrow x_{0}} u(x)=-\infty \quad\left(\forall x_{0} \in \Gamma_{\infty}\right),
$$

wobei $x$ innerhalb eines festen, sonst beliebigen $O_{\sigma_{1}}^{\sigma_{2}}$ gegen $x_{0}$ konvergiert. Man setze

$$
L\left(\sigma, \frac{u}{u_{2}}\right)=\sup _{x \in \Gamma_{\sigma}} \frac{u(x)}{u_{2}(x)}
$$

und nehme an, L sei endlich. Dann ist diese Größe eine konvexe Funktion von $\sigma$ in $J$. Es ist selbstverständlich, daß die Menge $\Gamma_{\infty}$ nicht beliebig sein kann. Sie unterliegt offenbar Bedingungen, die sichern, daß die Funktion $u_{1}$ in $G$ endlich ist und bei Annäherung an $\Gamma_{\infty}$ bzw. $\Gamma-\Gamma_{\infty}$ gegen $+\infty$ bzw. gegen Null konvergiert. Einfachere Fälle dieses Problems für die klassische Differentialgleichung (1.6) sind von de la VALLÉE-POUSSIN in einer fast vergessenen Note untersucht worden ${ }^{6}$.

Beweis des Satzes 4. Man setze für $x \in O_{\sigma_{1}}^{\sigma_{2}}$

$$
v=u-\left\{L_{1} \frac{\sigma_{2}-\omega}{\sigma_{2}-\sigma_{1}}+L_{2} \frac{\omega-\sigma_{1}}{\sigma_{2}-\sigma_{1}}\right\} u_{2}
$$

mit 


$$
L_{1}=L\left(\sigma_{1}, \frac{u}{u_{2}}\right), \quad L_{2}=L\left(\sigma_{2}, \frac{u}{u_{2}}\right)
$$

Dann gehört $v$ wegen

$$
v=u-\frac{L_{2}-L_{1}}{\sigma_{2}-\sigma_{1}} u_{1}+\frac{L_{2} \sigma_{1}-L_{1} \sigma_{2}}{\sigma_{2}-\sigma_{1}} u_{2}
$$

der Familie $A[G]$ an. Es sei jetzt $x_{0}$ ein Randpunkt von $O_{\sigma_{1}}^{\sigma_{2}}$. Ist $x_{0} \notin \Gamma_{\infty}$, so liegt $x_{0}$ in $G$, und somit gilt wegen

$$
\varlimsup_{x \rightarrow x_{0}} \frac{v(x)}{u_{2}(x)} \leq 0 \text {. }
$$

auch

$$
\varlimsup_{x \rightarrow x_{0}} v(x) \leq 0 .
$$

Ist $x_{0} \in \Gamma_{\infty}$, so bleibt bei Annäherung von $x \in O_{\sigma_{1}}^{\sigma_{2}}$ an $x_{0}$ die geschweifte Klammer rechts in (3.10) endlich und $u$ konvergiert gegen $-\infty$. Somit gilt (3. 11) für jeden Randpunkt $x_{0}$ von $O_{\sigma_{1}}^{\sigma_{2}}$ Es ist also $v(x) \leq 0$ in $O_{\sigma_{1}}^{\sigma_{2}}$ und somit

$$
\frac{u}{u_{2}} \leq L_{1} \frac{\sigma_{2}-\omega}{\sigma_{2}-\sigma_{1}}+L_{2} \frac{\omega-\sigma_{1}}{\sigma_{2}-\sigma_{1}}
$$

in $O_{\sigma_{1}}^{\sigma_{2}}$. Die Einschränkung von $x$ auf $\Gamma_{\sigma}$ und der Übergang zum Supremum liefern den Beweis des Satzes.

Ein entsprechender Satz gilt, wenn man $u>0$ in $G$ annimmt und (3. 8) durch die Bedingung

$$
\varlimsup_{x \rightarrow x_{1}} u(x) \leq 0 \quad\left(x_{1}, \in \Gamma_{\infty}\right)
$$

ersetzt. Wir überlassen den Beweis dem Leser.

SAtz 5. Es bezeichnen $u_{1}, u_{2}$ zwei Lösungen von (1.2) in $G$ mit den Eigenschaften:

1.

2.

3.

4.

5 .

$$
\begin{gathered}
u_{1}>0, \quad u_{2}>0 . \\
a=\inf _{x \in G} \omega(x)=0, \quad b=\sup _{x \in G} \omega(x)=+\infty .
\end{gathered}
$$$$
\left.\bar{O}_{\sigma_{1}}^{\sigma_{2}} \cap \Gamma_{0}=\phi, \quad \bar{O}_{\sigma_{1}}^{\sigma_{2}} \cap \Gamma_{\infty}=\phi \text { für jedes }\left[\sigma_{1}, \sigma_{2}\right] \subset J=\right] 0,+\infty[\text {. }
$$

4.

$$
\lim _{x \rightarrow x_{0}} u_{1}(x)=\infty \quad\left(\forall x_{0} \in \Gamma_{\infty}\right) .
$$

$$
\lim _{x \rightarrow x_{0}} u_{1}(x)=0 \quad\left(\forall x_{0} \in \Gamma-\Gamma_{\infty}\right) .
$$


6.

$$
\lim _{x \rightarrow x_{0}} u_{2}(x)=+\infty \quad\left(\forall x_{0} \in \Gamma_{0}\right) .
$$

7.

$$
\lim _{x \rightarrow x_{0}} u_{2}(x)=0 \quad\left(\forall x_{0} \in \Gamma-\Gamma_{0}\right) .
$$

Es sei $u \in A[G]$ und habe die Eigenschaft

$$
\varlimsup_{x \rightarrow x_{1}} u(x) \leq 0 \quad\left(\forall x_{1} \in \Gamma^{*}\right),
$$

wobei

$$
\Gamma^{*}=\cup\left\{\widetilde{O}_{\sigma_{1}}^{\sigma_{2}} \cap \Gamma:\right] \sigma_{1}, \sigma_{2}[\subset J\}
$$

ist. Man setze

$$
L\left(\sigma, \frac{u}{u_{2}}\right)=\sup _{x \in \Gamma_{\sigma}} \frac{u(x)}{u_{2}(x)}
$$

und nehme an, $L$ sei endlich. Dann ist $L$ eine konvexe Funktion von $\sigma$ in $J$.

Beweis. Man setze wieder für $x \in O_{\sigma_{1}}^{\sigma_{2}}$

$$
v=u-\left\{L_{1} \frac{\sigma_{2}-\omega}{\sigma_{2}-\sigma_{1}}+L_{2} \frac{\omega-\sigma_{1}}{\sigma_{2}-\sigma_{1}}\right\} u_{2} .
$$

Dann gehört $v$ der Klasse $A[G]$ an. Es sei $x_{0}$ ein Randpunkt von $O_{\sigma_{1}}^{\sigma_{2}}$. Ist $x_{0} \in \bar{O}_{\sigma_{1}}^{\sigma_{2}} \cap \Gamma$, so gilt wegen (3.13), sowie 6 und 7

$$
\varlimsup_{x \rightarrow x_{0}} \frac{v(x)}{u_{2}(x)} \leq 0
$$

und somit auch

$$
\varlimsup_{x \rightarrow x_{0}} v(x) \leq 0 .
$$

Ist dagegen $x_{0} \notin \bar{O}_{\sigma_{1}}^{\sigma_{2}} \cap \Gamma$, so kann $x_{0}$ entweder auf $\Gamma_{\sigma_{1}}$ oder $\Gamma_{\sigma_{2}}$ liegen. Das liefert wegen

$$
\varlimsup_{x \rightarrow x_{0}} \frac{u(x)}{u_{2}(x)} \leq L_{1} \quad\left(x_{0} \in \Gamma_{\sigma_{1}}\right)
$$

bzw.

$$
\varlimsup_{x \rightarrow x_{0}} \frac{u(x)}{u_{2}(x)} \leq L_{2} \quad\left(x_{0} \in \Gamma_{\sigma_{2}}\right)
$$

wieder die Ungleichungen (3.16) und (3.17). Mithin gilt die Ungleichung

$$
\frac{u(x)}{u_{2}(x)} \leq L_{1} \frac{\sigma_{2}-\omega(x)}{\sigma_{2}-\sigma_{1}}+L_{2} \frac{\omega(x)-\sigma_{1}}{\sigma_{2}-\sigma_{1}}
$$


und daher durch Einschränkung von $x$ auf $\Gamma_{\sigma}$

$$
L \leq L_{1} \frac{\sigma_{2}-\sigma}{\sigma_{2}-\sigma_{1}}+L_{2} \frac{\sigma-\sigma_{1}}{\sigma_{2}-\sigma_{1}}
$$

Das beweist den Satz 5.

Der Satz 5 stellt eine weitgehende Verallgemeinerung eines klassischen Satzes von PHRAGMÉN und LINDELÖF dar7) und zeigt gleichzeitig die Stärke der verwendeten Methode.

Wendet man die Begriffsbildungen und die Ergebnisse des Satzes 1 an, so erhält man leicht die Abschätzung

$$
L\left(\sigma, \frac{u}{u_{2}}\right) \leq C_{2}+C_{1} \sigma
$$

mit

$$
C_{2}=\lim _{\sigma \rightarrow 0} L\left(\sigma, \frac{u}{u_{2}}\right), \quad C_{1}=\lim _{\sigma \rightarrow+\infty} L\left(\sigma, \frac{u}{u_{1}}\right) .
$$

Die Abschätzung (3.20) ist von besonderem Interesse, falls $u$ die Bedingung (3. 13) für jeden Punkt $x_{0}$ des Randes $\Gamma$ erfüllt. In diesem Falle sind beide Konstanten $\leq 0$ und endlich, und man erhält wegen

$$
\frac{u(x)}{u_{2}(x)} \leq L\left(\sigma, \frac{u}{u_{2}}\right)
$$

die Ungleichung

$$
\frac{u(x)}{u_{2}(x)} \leq C_{2}+C_{1} \frac{u_{1}(x)}{u_{2}(x)}
$$

d.h.

$$
u(x) \leq C_{1} u_{1}(x)+C_{2} u_{2}(x) .
$$

Sind $C_{1}, C_{2}<0$, so liegt hier eine Verschärfung der Ungleichung $u(x) \leq 0$ vor. Sämtliche hier bewiesene Sätze gelten in entsprechender Form ( $\geq$ anstelle $\leq$ und inf statt sup), wenn man $u$ superharmonisch in $G, d . h . \quad u \in A^{\prime}[G]$ voraussetzt. Wir kommen in 6 darauf zurück.

4. Verallgemeinerung eines Satzes von Phragmén und Lindelöf. Es bedeute $C_{n}$ die offene Einheitskugel in $\boldsymbol{R}^{n}, d . h$. die Punktmenge

$\{x:\|x\|<1\}$. Wir legen die Potentialgleichung (1.6) zugrunde und beachten, daß die Funktion 


$$
u_{1}(x)=\left\{\begin{array}{l}
\log \|x\|^{-1} \quad(n=2) \\
\frac{1}{n-2}\|x\|^{-(n-2)} \quad(n>2)
\end{array}\right.
$$

in $\boldsymbol{R}^{n}-\{0\}$ der Differentialgleichung (1.6) genügt. Die Anwendung des Satzes 3 mit den Funktionen $u_{1}(x)+$ geeignete Konstante, $u_{2}=1$ führt ohne weiteres zu den Sätzen von HADAMARD.

Hat die Differentialgleichung (1.2) eine Grundlösung $l=[l(x, a)]$ in der Umgebung eines Punktes a von $\boldsymbol{R}^{n}{ }^{8)}$, so führt die Wahl $u_{1}=l$ und $u_{2}=$ reguläres Integral von (1.2) in der Umgebung von $a$, zu einem Satz vom Hadamardschen Typus.

Eine Reihe von Sätzen, die sämtlich Spezialfälle der Sätze 4 und 5 (Sätze vom Phragmén-Lindelöfschen Typus) sind, erhält man, wenn man die Funktionen

$$
h\left(x, x_{0}\right)=\frac{1-\|x\|^{2}}{\left\|x-x_{0}\right\|^{n}} \quad\left(\left\|x_{0}\right\|=1\right)
$$

zugrundelegt, die in $C_{n}$ der Differentialgleichung (1.6) genügen.

Folgender Satz ist eine unmittelbare Folge des Satzes 4.

Satz 6. Es sei $x_{0}$ ein (fester) Punkt des Randes $\Gamma$ von $C_{n}$ und $u$ eine in $C_{n}$ subharmonische Funktion, welche der Bedingung

$$
\lim _{x \rightarrow x_{0}} u(x)=-\infty
$$

genügt, sofern $x$ innerhalb jeder Menge

$$
O_{\sigma}^{\infty}=\left\{x: \sigma<h\left(x, x_{0}\right)<+\infty\right\}
$$

gegen $x_{0}$ konvergiert. Man definiere $\Gamma_{\sigma}$ durch die Gleichung

$$
\Gamma_{\sigma}=\left\{x: h\left(x, x_{0}\right)=\sigma, \sigma \in\right] 0,+\infty[\}
$$

und setze

$$
L(\sigma, u)=\sup _{x \in \Gamma_{\sigma}} u(x) .
$$

Dann ist $L$ eine konvexe Funktion von $\sigma$ in $] 0,+\infty[$.

Gilt darüber hinaus die Ungleichung

$$
\varlimsup_{x \rightarrow x_{1}} u(x) \leq 0 \quad\left(x_{1} \in \Gamma-\left\{x_{0}\right\}\right),
$$

wobei $\Gamma$ der Rand von $C_{n}$ ist, so gilt

$$
u(x) \leq C_{0} h\left(x, x_{0}\right)
$$


in $C_{n}$ mit

$$
C_{0}=\lim _{\sigma \rightarrow+\infty}\left\{\sup _{x \in \Gamma_{\sigma}} \frac{u(x)}{h\left(x, x_{0}\right)}\right\}
$$

Der Beweis dieses Satzes kann ohne weiteres nach dem Muster des Beweises des Satzes 4 sowie der Entwicklungen von 2 vom Leser selbst erbracht werden. Nachfolgender Satz kann als die wichtigste Anwendung des Satzes 5 angesehen werden:

Satz 7. Die Funktion $u$ sei in $C_{n}$ subharmonisch und $x_{0}$ ein fester Punkt des Randes $\Gamma$ von $C_{n}$. Ferner sei u subharmonisch in $C_{n}$ und genüge dort der Ungleichung

$$
\varlimsup_{x \rightarrow x_{1}} u(x) \leq 0
$$

für jeden Punkt $x_{1}$ von $\Gamma \neq x_{0}$. Man setze

$$
\omega(x)=\frac{\left\|x+x_{0}\right\|^{n}}{\left\|x-x_{0}\right\|^{n}}=\frac{h\left(x, x_{0}\right)}{h\left(x,-x_{0}\right)},
$$

und definiere $\Gamma_{\sigma}$ durch die Gleichung

$$
\Gamma_{\sigma}=\left\{x: x \in C_{n}, \frac{\left\|x+x_{0}\right\|}{\left\|x-x_{0}\right\|}=\sigma, \sigma \in\right] 0,+\infty[\} .
$$

Setzt man dann

$$
L(\sigma, u)=\sup _{x \in \Gamma_{\sigma}}\left\{u(x)-\frac{\left\|x+x_{0}\right\|^{n}}{1-\|x\|^{2}}\right\}=\sup _{x \in \Gamma_{\sigma}}\left\{\frac{u(x)}{h\left(x,-x_{0}\right)}\right\},
$$

so hat $L(\sigma, u)$ die Eigenschaften:

(1) $L(\sigma, u)$ ist endlich.

(2) L ist $\sigma^{n}$-konvex in $\left.J=\right] 0,+\infty[$.

Der Beweis dieses Satzes erfordert eine Wiederholung des Beweises des Satzes 5 und wird hier nicht explizit angegeben ${ }^{9}$.

Aus dem Satz 7 folgt unter Heranziehung der Ergebnisse von 3, daß die Grenzwerte

$$
C\left(x_{0}\right)=\lim _{\sigma \rightarrow+\infty}\left\{\sup _{x \in \Gamma_{\sigma}} \frac{u(x)}{h\left(x, x_{0}\right)}\right\}=\varlimsup_{x \rightarrow x_{0}}\left\{\frac{u(x)}{h\left(x, x_{0}\right)}\right\}
$$

und

$$
C\left(-x_{0}\right)=\lim _{\sigma \rightarrow 0}\left\{\sup _{x \in \Gamma_{\sigma}} \frac{u(x)}{h\left(x,-x_{0}\right)}\right\}=\varlimsup_{x \rightarrow-x_{0}}\left\{\frac{u(x)}{h\left(x,-x_{0}\right)}\right\}
$$


existieren (ein Ergebnis, das den Inhalt des klassischen Phragmén-Lindelöfschen Satzes als Spezialfall enthält), und daß (falls $C\left(x_{0}\right)$ endlich ist) die Ungleichung

$$
u(x) \leq C\left(-x_{0}\right) h\left(x,-x_{0}\right)+C\left(x_{0}\right) h\left(x, x_{0}\right),
$$

d.h.

$$
u(x) \leq C\left(-x_{0}\right) \frac{1-\|x\|^{2}}{\left\|x+x_{0}\right\|^{n}}+C\left(x_{0}\right) \frac{1-\|x\|^{2}}{\left\|x-x_{0}\right\|^{n}}
$$

gilt.

Die Sätze 5 und 6 lassen sich insofern verallgemeinern, als man dort die Funktion $h\left(x,-x_{0}\right)$ durch $h\left(x, x_{1}\right)\left(\left\|x_{1}\right\|=1, x_{1} \neq x_{0}\right)$ ersetzen kann. Auch kann man anstelle $C_{n}$ den Halbraum

$$
H_{n}=\left\{x: x \in \boldsymbol{R}^{n}, x_{1}>0\right\}
$$

zugrunde legen und anstelle der Funktionen $h\left(x, x_{0}\right), h\left(x,-x_{0}\right)$ die Funktionen $x_{1}$ bzw. $\frac{x_{1}}{\|x\|^{n-1}}$ nehmen. Der Leser findet die entsprechenden Entwicklungen an anderer Stelle (DINGHAS [6]) ausführlicher dargestellt.

5. Heranziehung des Randverhaltens. Die Entwicklungen der vorherigen Nummern können dazu verwendet werden, durch Heranziehung des Randverhaltens einer in $C_{n}$ subharmonischen Funktion $u$ die klassischen Aussagen über deren Anwachsen zu verschärfen.

Definition 3. Es sei u subharmonisch in $C_{n}$ und genüge dort der Bedingung

$$
\varlimsup_{x \rightarrow x_{0}} u(x) \leq 0
$$

für jeden Punkt $x_{0}$ des Randes $\Gamma$ von $C_{n}$. Wir setzen

$$
-\alpha\left(x_{0}\right)=\varlimsup_{x \rightarrow x_{0}}\left\{\frac{u(x)}{h\left(x, x_{0}\right)}\right\} .
$$

Dann soll $E$ (ausführlicher $E_{n}$ ) durch die Gleichung

$$
E=\left\{x_{0}: x_{0} \in \Gamma, \alpha\left(x_{0}\right)>0\right\}
$$

definiert werden.

Die Definition der Menge $E$ für eine in $C_{n}$ subharmonische Funktion, die dort der Bedingung

$$
\varlimsup_{x \rightarrow x_{0}} u(x) \leq M<+\infty
$$

genügt, lautet entsprechend. 
SAtz 8. Es sei u subharmonisch in $C_{n}$ und genüge dort der Bedingung

$$
\varlimsup_{x \rightarrow x_{0}} u(x) \leq 0
$$

für jeden Punkt des Randes $\Gamma$ von $C_{n}$. Dann gilt folgendes:

(1) $E$ ist leer, endlich oder abzählbar.

(2) Die Summe

$$
\sum_{y \equiv E} \alpha(y)
$$

ist (falls $E$ abzählbar ist) konvergent.

(3) Es gilt die Ungleichung

$$
u(x) \leq-\sum_{y \in E} \alpha(y) h(x, y) \quad\left(x \in C_{n}\right) .
$$

Berveis. Es seien $y_{1}, \ldots \ldots, y_{q} \quad q$ Punkte von E. Dann genügt

$$
v(x)=u(x)+\sum_{k=1}^{q} \alpha\left(y_{k}\right) h\left(x, y_{k}\right)
$$

der Ungleichung (5.1) für jeden Punkt $x_{0}$ von $\Gamma$. Daraus folgt zunächst $v(x) \leq 0$ in $C_{n}$. Nun liefert eine leichte Abschätzung von $h\left(x, x_{0}\right)$ nach unten die Ungleichung

$$
u(x) \leq-\frac{1-\|x\|^{2}}{(1+\|x\|)^{n}} \sum_{k=1}^{q} \alpha\left(y_{k}\right)
$$

Diese Ungleichung hat wiederum zur Folge (da $u$ generell $\neq-\infty$ angenommen wird), dass jede der Mengen

$$
\{y: y \in \Gamma, 0<p \leq \alpha(y) \leq q<+\infty\}
$$

bei festen $p, q$ höchstens endlich sein kann. Das beweist (1). Man schreibe jetzt $l(x)$ für die durch die Gleichung (4.1) definierte Funktion $u_{1}(x)$ und definiere die Greensche Funktion $g(x, a)(\|\mid a\|<1)$ von $C_{n}$ durch die Gleichung

$$
g(x, a)=l(x-a)-l\left(\frac{a}{a-\|a\|^{2} x}\right) .
$$

Definition 4. Es sei $u$ subharmonisch in $C_{n}$. Dann soll für jedes $a \in C_{n}-\gamma(a)$ die Größe

$$
\lim _{r \rightarrow 0}\left\{\sup _{\|x-a\|=r} \frac{u(x)}{l(x-a)}\right\}
$$


bedeuten.

Offenbar ist

$$
-r(a)=\lim _{r \rightarrow 0}\left\{\sup _{|| x-a \|=r} \frac{u(x)}{g(x, a)}\right\} \text {. }
$$

$\mathrm{Daß}$ der Grenzwert $-\gamma(a)$ existiert, ist eine Folgerung des klassischen Hadamardschen Satzes für subharmonische Funktionen in $C_{n}$ und kann ohne Schwierigkeit auf Grund der Entwicklungen von 3 bewiesen werden.

Nachfolgender Satz sei hier ohne Beweis angeführt:

SATz 9. Es sei u subharmonisch in $C_{n}$ und genüge dort der Bedingung

$$
\varlimsup_{x \rightarrow x_{0}} u(x) \leq 0
$$

für jeden Punkt $x_{0}$ des Randes $\Gamma$ von $C_{n}$.

Man definiere die Teilmenge $E_{0}$ von $C_{n}$ durch die Gleichung

$$
E_{0}=\left\{a: a \in C_{n}, \quad r(a)>0\right\} \text {. }
$$

Dann gilt folgendes:

(1) $E_{0}$ ist leer, endlich oder abzählbar.

(2) Die Summe

$$
\sum_{a \in E_{0}} \gamma(a)(1-\|a\|)
$$

ist (falls $E_{0}$ abzählbar ist) konvergent.

(3) Es ist

$$
u(x) \leq-\sum_{a \in E_{0}} \gamma(a) g(x, a)
$$

Den Beweis dieses Satzes findet der Leser in meiner unter [5] zitierten Arbeit. Dort wird auch ein ausführlicher Beweis gegeben, daß man aus (5.16) die Ungleichung

$$
u(x) \leq-\frac{1}{q} \frac{1-\|x\|^{q}}{(1+\|x\|)^{2 q}} \sum_{a \in E_{0}} \gamma(a)(1-\|a\|)
$$

mit $q=\max \{1, n-2\}$ ableiten kann.

Die Verbindung der Sätze 8 und 9 liefert unter der Voraussetzung (5. 13) die Ungleichung

(5. 18) $\quad u(x) \leq-\frac{1}{q} \frac{1-\|x\|^{q}}{(1+\|x\|)^{2 q}} \sum_{a \in E_{0}} \gamma(a)(1-\|a\|)-\sum_{y \in E} \alpha(y) \frac{1-\|x\|^{2}}{\|x-y\|^{n}}$. 
Diese liefert eine Verallgemeinerung eines Satzes von BLASCHKE10) für holomorphe Funktionen in $C_{n}$, der in der nächsten Nummer besprochen wird.

6. Nochmals die Sätze von Phragmén-Lindelöf und Blaschke. Der Satz von Julia-Wolff-Garathéodory. Der Fall $n=2$ ist insofern interessant als die Zugrundelegung der durch den Punkt $z=\infty$ kompaktifizierten komplexen Ebene eine Reihe von Anwendungen der in 3, 4 und 5 bewiesenen Sätze gestattet. Hier sollen lediglich drei der in Frage kommenden Sätze behandelt werden.

Es bezeichne $z=x+i y(x, y$ reell $)$ einen Punkt von $C_{2}$ und $w=[w(z)]$ eine in $C_{2}$ holomorphe Funktion mit der Eigenschaft

$$
\varlimsup_{z \rightarrow \zeta}|w(z)| \leq 1
$$

für jeden Randpunkt $\zeta \neq 1$. Man setze

$$
\sigma=\left|\frac{1+z}{1-z}\right|
$$

und bezeichne bei gegebenem $\sigma$ den Durchschnitt von $C_{2}$ mit der Kreisperipherie (6. 2) durch $\Gamma_{\sigma}$. Wird dann

$$
L=L(\sigma, w)=\sup _{z \in T_{\sigma}}\left\{\log |w(z)| \frac{|1+z|^{2}}{1-|z|^{2}}\right\}
$$

gesetzt, so ist L endlich (DINGHAS [3]) und im Sinne der Ungleichung (2.5) konvex in bezug auf $\sigma^{2}$. Ferner existiert der Grenzwert

$$
\lim _{\sigma \rightarrow+\infty}\left\{\sup _{z \in \Gamma_{\sigma}}\left(\log |w(z)| \frac{|1-z|^{2}}{1-|z|^{2}}\right)\right\}
$$

und ist entweder gleich $+\infty$ oder gleich einer endlichen Zahl.

Dieses Ergebnis stellt lediglich eine Umformulierung des klassischen PhragménLindelöfschen Satzes dar. Dessen Verallgemeinerung auf Punktmengen (auf $\Gamma$ ) von der Kapazität Null stößt auf keine wesentlichen Schwierigkeiten. Die in 5 für jedes $n \geq 2$ bewiesenen Sätze 8 und 9 führen im vorliegenden Fall zu dem Satz:

SATz 10. Die (nichtkonstante) Funktion $w$ sei holomorph in $C_{2}$ und genüge der Ungleichung (6. 1). Es bezeichne $E_{0}$ die Menge der Nullstellen a von $w$ in $C_{2}$ und $E$ die (höchstens abzählbare) Menge der Randpunkte $\zeta$ von $C_{2}$ mit der Eigenschaft

$$
-\alpha(\zeta)=\varlimsup_{z \rightarrow \zeta}\left\{\log |w(z)| \frac{|\zeta-z|^{2}}{1-|z|^{2}}\right\}>0 \text {. }
$$


Dann gilt die Ungleichung

$$
|w(z)| \leq \prod_{a \in E_{0}}\left|\frac{z-a}{1-\bar{a} z}\right| \cdot \exp \left\{-\sum_{\zeta \in E} \alpha(\zeta) \frac{1-|z|^{2}}{|\zeta-z|^{2}}\right\} .
$$

Wegen

$$
\left|\frac{z-a}{1-\bar{a} z}\right| \leq \exp \left\{-\frac{1}{2}(1-|a|)(1-|z|)\right\},
$$

kann (DINGHAS [5]) diese Ungleichung auch in der Form

$$
|w(z)| \leq \exp \left\{-\frac{A}{2}(1-|z|)\right\}
$$

mit

$$
A=\sum_{a \in E_{0}}(1-|a|)+\sum_{\zeta \in E} \alpha(\zeta)
$$

geschrieben werden. Daß $A$ endlich sein muß, ist mit Rücksicht auf die gemachte Voraussetzung, $w \neq$ Konstante, selbstverständlich und bedarf hier keiner weiteren Erläuterung.

Der Fall einer in $C_{n}$ superharmonischen Funktion, die für jeden Randpunkt $x_{1} \neq x_{0}\left(\left\|x_{1}\right\|=\left\|x_{0}\right\|=1\right)$ von $C_{n_{0}}$ der Ungleichung

$$
\lim _{x \rightarrow x_{1}} u(x) \geq 0
$$

genügt, führt durch Heranziehung der Funktion $-u$ zu einigen Sätzen, von denen wohl der interessanteste der klassische Satz von Julia-Wolff-Carathéodory sein dürfte.

Satz 11. Es sei u superharmonisch in $C_{n}$ und genüge dort der Ungleichung (6.8). Man definiere $\omega$ und $\Gamma_{\sigma}$ durch (4.11) bzw. (4. 12) und setze

$$
D(\sigma, u)=\inf _{x \in \Gamma_{\sigma}}\left\{u(x) \frac{\left\|x+x_{0}\right\|^{n}}{1-\|x\|^{2}}\right\} .
$$

Dann ist D im Sinne der Ungleichung

$$
D \geq D_{1} \frac{\sigma_{2}^{n}-\sigma^{n}}{\sigma_{2}^{n}-\sigma_{1}^{\prime \prime}}+D_{2} \frac{\sigma^{n}-\sigma_{1}^{n}}{\sigma_{2}^{n}-\sigma_{1}^{n}}
$$

konkav in bezug auf $\sigma^{n}$, und es gilt die Ungleichung

$$
u(x) \geq D\left(x_{0}\right) \frac{1-\|x\|^{2}}{\left\|x-x_{0}\right\|^{n}}
$$

mit 


$$
D\left(x_{0}\right)=\lim _{\sigma \rightarrow+\infty} D(\sigma, u) \quad\left(0 \leq D\left(x_{0}\right)<+\infty\right) .
$$

Die Heranziehung der in $C_{2}$ holomorphen Funktion $w$, die dort der Ungleichung (6. 1) genügt, führt, indem man $u$ gleich

$$
\frac{1-|w|^{2}}{|\zeta-w|^{2}} \quad(\zeta \text { fest }, \quad|\zeta|=1)
$$

nimmt, direkt zu dem Julia-Wolff-Carathéodoryschen Satz. In der Tat liefert (6. 11) die Ungleichung

$$
\frac{1-|w(z)|^{2}}{|\zeta-w(z)|^{2}} \geq D(\zeta) \frac{1-|z|^{2}}{|\zeta-z|^{2}}
$$

mit $0 \leq D(\zeta)<+\infty$. Die Bedingungen dafür, daß $D(\zeta)>0$ ist (die eigentlich den Inhalt des Satzes von JULIA-WOLFF-CARATHÉODORY ausmachen), werden uns hier insofern nicht beschäftigen, als die Arbeit von CARATHÉODORY, sofern man (6. 12) zugrundelegt ${ }^{11)}$, das gesamte Beweismaterial in großer Ausführlichkeit liefert.

7. Offene Fragen. Die in den Nummern 4, 5 und 6 gegebenen Anwendungen der allgemeinen Sätze 3,4 und 5 könnten vermehrt werden, wenn man neben dem Nachweis der Existenz einer Grundlösung von (1.2) noch den Existenzbeweis einer der Evans-Selbergschen Kapazitätsfunktion von (1. 6) entsprechenden Funktion von (1.2) erbringen könnte. Dabei würde es sich um Lösungen von (1.2) in $G$ handeln, die bei Annäherung an eine kompakte Teilmenge $K$ des Randes $\Gamma$ von $G$ gegen $+\infty$ strebt, während sie auf $\Gamma-K$ die Randwerte Null haben. Die vorhin zitierte Note von de la Vallée Poussin sei hier nochmals erwähnt. Selbstverständlich braucht man, um konkretere Resultate zu erzielen, nicht bloß die Existenz solcher Funktionen zu erbringen, sondern noch deren ausdrucksmäßige Beherrschung. Zur Zeit könnte man solche Überlegungen höchstens für die von PICARD (PICARD [1]) erstmalig studierten partiellen Differentialgleichungen vom elliptischen Typus in der kanonischen Form

$$
\Delta u+\sum_{k=1}^{n} a_{k} u_{x_{k}}+c u=0
$$

mit analytischen Koeffizienten und für die Dimension $n=2$ anstellen ${ }^{12)}$. Im allgemeinen Fall jedoch sind die Schwierigkeiten, auf die man schon bei der Konstruktion der Greenschen Funktion stößt, so groß, daß die Hoffnung 
eines weiteren Vordringens auf diesem Wege mehr oder weniger gering erscheint.-

\section{FUßNOTEN}

1) Zur allgemeinen Orientierung vgl. man COURANT [1], S. $320 \mathrm{ff}$.

2) Courant [1], S. 326.

3) Diesse Fassung des HOPFSCHEN Maximumprinzips ist schärfer als die entsprechende Formulierung bei COURANT (COURANT [1], S. 326).

4) Die hier zugrundegelegte Definition der subharmonischen Funktion ist allgemeiner als diejenige bei COURANT (COURANT [1], S. 342).

5) Für die Differentialgleichung (1.6) sowie für sämtliche elliptischen Differentialgleichungen mit c $=0$ kann als $u_{2}$ die Konstante 1 genommen werden.

6) DE LA VALLÉE POUSSIN [1].

7) Die Literatur über den Phragmén-Lindelöfschen Satz ist sehr groß. Was der Leser an Zusammenhängen braucht, findet er in: DINGHAS [1], [3], [4] und [6].

8) Zur Begriffsbildung vgl. man neben COURANT (COURANT [1]) noch das historisch wertvolle Buch von PICARD (PICARD [1]). Allgemein kann man jede Lösung von (1.2) in einer punktierten Umgebung von a, die bei Annäherung an a gegen $+\infty$ konvergiert, als eine Grundlösung von (1.2) bezeichnen. Diese ist dann bis auf eine multiplikative Konstante definiert.

9) Man vgl. DINGHAS [2], [4] und [6].

10) Man vgl. BLASCHKE [1].

11) Neben CARATHÉODORY [1] vgl. man JULIA [1] und WOLFF [1], [2]. Darüber hinaus auch TSUJI [1] und [2] sowie DINGHAS [1] und [2].

12) Man vgl. etwa PICARD [1] sowie COURANT [1].

\section{LITERATUR}

BLASCHKE, W.: [1] Über eine Erweiterung des Satzes von Vitali über Folgen analytischer Funktionen. Leipz. Ber. 67, 1915, 194-200.

CARATHÉODORY, C.: [1] Über die Winkelderivierten von beschränkten analytischen Funktionen. Sitzungsber. Preuss. Akad. d. Wissensch. Phys.-Math. Klasse, 1929, 34-54.

COURANT, R., HILBERT, D.: [1] Methods of Mathematical Physics, Vol. II, (Partial Differential Equations), Interscience Publishers, New-York-London, 1962.

DINGHAS, A.: [1] Über das Phargmén-Lindelöfsche Prinzip und den Julia-Carathéodoryschen Satz. Sitzungsber. d. Preuß. Akad. Wiss., Phys.-Math. Klasse, 1938, 32-48.

DINGHAS, A.: [2] Über positive harmonische Funktionen in einem Halbraum. Math. Zeitschr. 46, 1940, 559-570.

DINGHAS, A.: [3] Über das Anwachsen einiger Klassen von subharmonischen und verwandten Funktionen. Ann. Acad. Sci. Fenn. Ser. A I No. 336/1, 1963, 27 S.

DINGHAS, A.: [4] Über einige Konvexitätsfragen bei partiellen Differentialgleichungen vom Sturmschen Typus. Math. Ann. 155, 1964, S. 397-421.

DINGHAS, A.: [5] Über den Einfluß von Unendlichkeitsstellen auf das Wachstum subharmonischer Funktionen im $E^{n}$. Monatsh. f. Math. 68, 1964, 289-306.

DINGHAS, A.: [6] Über einige Konvexitätssätze für die Mittelwerte von subharmonischen Funktionen. Journ. d. Math. pures et appl. (9), 44, 1965, S. 223-247.

JULIA, G.: [1] Extension d'un Lemme de Schwarz, Acta Math. 42, 1920. 349-355.

PICARD, E.: [1] Leçons sur quelques types simples d'équations aux derivées partielles. 
Paris: Gauthier-Villars, 1927.

TSUJI, M.: [1] On a positiv harmonic function in a half plane. Jap. Journ. Math. 15, 1939, 277-285.

TSUJI, M.: [2] Potential Theory in Modern Functions Theory. Tokyo: Maruzen Co., Ltd. 1959.

VALLÉE POUSSIN, CH. de la: [1] Expression nouvelle d'une fonction harmonique positive dans une aire et nulle en tout autre point du bord sauf un. Annal. Soc. Bruxelles, A 53, 113-122. WOLFF, J.: [1] Démonstration d'un théorème sur la conservation des angles dans la représentation conforme d'un domaine au voisinage du'n point frontière. Proc. Akad. Amsterdam 38, 1935, 46-50.

WOLFF, J.: [2] Sur la réprésentation conforme des bandes. Comp. Math. 1, 1935, 217222. 